\title{
ACERCAMIENTO AL LENGUAJE TÉCNICO ALEMÁN EN EL CAMPO DE LA AUTOMOCIÓN
}

\author{
Alfonso Corbacho Sánchez
}

Universidad de Extremadura

\section{INTRODUCCIÓN}

Desde hace unas décadas, el mercado de la automoción se erige como uno de los colosos de la economía mundial. Y en este paraje histórico brilla con luz propia la representación automovilística de Alemania, país con un peso específico incuestionable en materia de motores, y no sólo en Europa donde lidera gran parte del mercado, sino incluso a escala mundial.

Nos hallamos en una sociedad que evoluciona a un ritmo ciertamente vertiginoso y como consecuencia inmediata de todo ello nos encontramos con representaciones lingüísticas que tampoco se quedan desfasadas, acoplándose, por tanto, al correr de los tiempos y ajustándose a las necesidades que las circunstancias exigen.

Tomando el marco automovilístico como punto de referencia y como ámbito de investigación, es objeto de reciente atención la producción de un léxico especializado procedente del sector de la automoción. Desde un punto de vista lingüístico, las investigaciones distan aún mucho de alcanzar una formulación coherente, pero, en líneas generales, se comienza a vislumbrar y acotar una serie de características de lo que puede comenzar a denominarse "lenguaje técnico de la automoción"1. De este modo, la constitución de un lenguaje especializado en este sector, utilizado como moneda de cambio tanto en círculos formativos como profesionales, nos hace concebir esperanzas de que no se trata de una quimera, más bien de una realidad eminente.

No obstante, respetando nuestro planteamiento inicial, una definición del lenguaje técnico alemán en el ámbito de la automoción, desde una perspectiva teórica, continúa siendo un desideratum. La tarea de desarrollar y explicitar un concepto se hace cada vez más urgente, cuando el número de usuarios de una disciplina experimenta un aumento de considerables proporciones. En esta situación, llama de manera especial la atención el hecho de que, hasta el momento, no se hayan presentado trabajos de investigación rigurosos y exhaustivos sobre este lenguaje especializado, al menos no equiparable a otros lenguajes especializados. Quizás uno de los motivos de esta escasa atención investigadora esté centrado en que el alemán de la mecánica no se considere un lenguaje especializado con entidad propia frente a otros más tradicionales como el lenguaje jurídico o económico. Sin embargo, este

\footnotetext{
${ }^{1}$ No insistiremos en que nuestro trabajo queda enfocado desde la perspectiva del idioma alemán. Por otra parte, la flexibilidad que nos permite el presente campo de estudio, puede conducir a encontrar otras denominaciones para referirnos a la mismo realidad, como por ejemplo: "el lenguaje de la mecánica de automóviles".
} 
lenguaje específico es una disciplina lingüística ${ }^{2}$ relativamente joven que, actualmente, pugna por hacerse un hueco en el vasto campo de los lenguajes especializados.

En definitiva, pretendemos con esta contribución definir y delimitar el lenguaje técnico alemán de la automoción, y, por consiguiente, dar un paso hacia delante en el estudio de este campo del conocimiento, ubicándonos en el contexto de la Germanística española.

\section{El LENGUAJE TÉCNiCO de LA AUTOMOCIÓN}

Consideramos innecesario detenernos en cuestiones puramente sociológicas sobre la enorme repercusión de este fenómeno cotidiano. El automovilismo y la automoción han adquirido tal protagonismo y eco popular que hasta el más profano en este campo específico del saber se atreve a emitir un juicio valorativo sobre ciertos aspectos de esta materia. Gran parte de esta culpa la encontramos en los medios de comunicación y en la publicidad de la prensa y demás revistas especializadas.

Sin duda alguna, como ya hemos constatado, es Alemania el principal país de la Unión Europea en lo que respecta a ingeniería y diseño de automóviles. Y en este contexto la magia que genera el parque automovilístico en la actualidad ofrece como resultado a uno de los sectores industriales de mayor peso de la economía mundial. Pero esta explosión de creatividad no es exclusiva del mundo del automóvil, sino también de las terminologías y los lenguajes especiales. El lenguaje técnico de la automoción está directamente relacionado con los arrolladores progresos de la técnica que incorpora día tras día mecanismos cada vez más sofisticados. Por tanto, este lenguaje especializado evoluciona con el nuevo siglo y las nuevas tendencias que irrumpen en el mercado. Técnicos y profesionales pugnan constantemente por crear términos propios que marquen diferencias frente a sus competidores más directos ante el aluvión de nuevos modelos que no cesan de salir al mercado. Por ello, este vocabulario específico resulta ser determinante y decisivo para habituarnos al entorno técnico del momento, sí bien es cierto que los expertos no siempre utilizan expresiones sencillas y conceptos de fácil comprensión, aunque no cabe la menor duda, que el automóvil es un innegable elemento de progreso, y como tal, seguiremos asistiendo con toda seguridad a una imparable y desmesurada evolución terminológica en el sector automovilístico.

Asimismo, damos cuenta de que esta evolución terminológica es incapaz de ser secundada por los diccionarios especializados, por muy reciente que sea la publicación, dado el enorme caudal léxico que no interrumpe la incesante actividad de incrementar sus límites a un ritmo tan precipitado que no conoce precedentes. No debemos olvidar, que la continua y constante actualización del lenguaje técnico en materia automovilística favorece la proliferación de neologismos.

Con todo, no es objeto de esta publicación teorizar sobre el comcepto de los lenguajes especiales desde una perspectiva generalizada y exponer el amplio abanico de definiciones que nos ha aportado la investigación al respecto. Por ello, en un intento de sintetizar el concepto de lenguaje técnico, nos atrevemos a afirmar que es la utilización de la lengua en un campo profesional determinado. Así lo expone Hahn (1980:390) cuando indica que los lenguajes especiales "dienen in erster Linie der Kommunikation innerhalb von im weitesten

\footnotetext{
${ }^{2}$ Sí nos atrevemos a certificar la existencia de algunos trabajos sobre este fenómeno lingüístico. En la mayoría de los casos se han realizado desde la perspectiva de la traducción especializada, así como también desde el punto de vista de las terminologías.
} 
Sinne technisch und wissenschaftlich orientierten Handlungs- und Arbeitssystemen", incluyendo el lenguaje técnico de la automoción en el amplio grupo de los lenguajes técnicos.

Pero sí es nuestra intención presentar esta exposición de los lenguajes especiales desde el ángulo de la técnica y siempre referido al campo de la automoción, y en este sentido señala Arnzt/Picht la tan estrecha relación existente entre el lenguaje tecnológico y el lenguaje común (1995:39), concretamente en lo que se refiere al automóvil, cuando numerosas expresiónes en un principio técnicas terminan por asentarse en el lenguaje coloquial. También el lingüista Fluck (1976:36) hace referencia al lenguaje técnico de la automoción, cuando cita el estudio de $\mathrm{Kutzelnigg}^{3}$ en torno a la extensa variedad de significados del concepto Benzin, poniendo de relieve la precisión con la que opera el término, según el campo especializado en el que es utilizado por los diferentes colectivos profesionales.

En un intento de dar cumplida respuesta a nuestro planteamiento, partiremos de la tradicional cita del estudioso Hoffmann (1985: 2):

"Fachsprache - das ist die Gesamtheit aller sprachlichen Mittel, die in einem fachlich begrenzbaren Kommunikationsbereich verwendet werden, um die Verständigung der dort tätigen Fachleute zu gewährleisten".

Pues bien, tomando estas palabras como punto de referencia, consideraremos el lenguaje técnico de la automoción como el lenguaje técnico utilizado en la comunicación especializada por los profesionales pertenecientes al ámbito de la mecánica de motores, que se caracteriza por el uso de una terminología especializada y que manifesta unos rasgos lingüísticos particulares. Lógicamente, la aclaración es relativa a la lengua alemana, aunque podemos aplicarla a cualquier otra lengua.

\section{LA TRADUCCIÓN ESPECIALIIZADA}

No es ninguna novedad que hoy en día la traducción especializada domine con cierta autoridad el mercado profesional de los traductores. La proyección internacional de las relaciones comerciales se convierten en el responsable directo de que este tipo de traducción cobre día tras día mayor importancia. Frente al desafío que supone la tentativa de ofrecer una definición de la traducción técnica, somos conscientes de que articular una definición infalible resulta tarea imposible y más aún en un campo tan correoso como éste que requiere continuas matizaciones. No obstante, a grandes rasgos podemos definir la traducción técnica como toda traducción que intenta pasar de la lengua original a la lengua terminal el sentido integro de un texto específico de características técnicas.

Atendiendo a esta peculiaridad, la figura del traductor debe mostrar un perfecto dominio de la lengua origen como de la lengua meta, además de ser un especialista en el correspondiente campo del conocimiento objeto de traducción. Así, lo perfila Bachmann (1992: 51) al mantener "da $\beta$ die fachliche Komponente in vielen Fällen ausschlaggebend ist".

Por tanto, en lo tocante a la traducción especializada disponemos de elementos de juicio suficientes para mantener que este tipo de traducción se reserva a los especialistas en las

${ }^{3}$ Véase a Kutzelnigg, A. (1967), "Zur sprachlichen Bewältigung eines technischen Stoffbereichs". En: Lebende Sprachen 12. págs. 65-71.. 
diferentes áreas del conocimiento, concretamente en lo que respecta al dominio de las dos lenguas, tanto la lengua original y la lengua terminal, así como también en lo que concierne a la terminología específica. Naturalmente, que reunir todas estas facultades en un traductor técnico suelen ser casos bastante aislados y muy puntuales. Roelcke (1999: 146) se manifiesta en términos similares, cuando plantea las cinco competencias profesionales que debe reunir el traductor especializado:

"Erstens die allgemeinsprachliche Kompetenz der Ausgangs- und zweitens diejenige der Zielsprache im allgemeinen; drittens die fachsprachliche Kompetenz der Ausgangs- und viertens diejenige der Zielsprache im besonderen sowie fünftens eine hinreichende Sachkompetenz in dem betreffenden Fachbereich."

Curiosamente, Newmark (1987: 213), una de las autoridades en el campo de la traducción, en su libro Manual de traducción parece no conceder excesiva importancia a la formación de los diferentes campos específicos. Y lo expone en los términos siguientes:

“... para traducir un texto no se necesita ser un experto en la tecnología y el vocabulario pertinentes, basta con entenderlo y conocer por el momento el vocabulario que usa."

Por otra parte, y para finalizar este apartado, hacemos mención de otra de las cuestiones que se deben tener presentes en la traducción especializada, nos referimos al reducido margen, por no decir inexistente, de matices afectivos que debe contener el texto. El traductor especializado tiene que ajustarse rigurosamente a la terminología que oferte la lengua original.

\section{ANÁLISIS LINGÜÍSTICO Y TRADUCCIÓN DEL TEXTO}

El análisis lingüístico del discurso técnico de la automoción en lengua alemana nos brinda la posibilidad de verificar los distintos elementos morfosintácticos y léxicosemánticos que confluyen en este tipo de reproducciones lingüísticas. El texto, que sirve de apoyo a cuanto se expone en este análisis, revela una rigurosa naturaleza mecánica y corresponde a un artículo de Autofachmann, revista especializada en la formación de mecánicos de automóviles en centros de formación profesional. Antes de entrar en materia, queremos hacer una breve referencia en lo que afecta al texto. Obviamente, éste no reúne todas las características del lenguaje técnico en este campo del conocimiento, pero sí puede considerarse representativo dentro de los límites naturales de su extensión por agrupar las características más generales y comunes en todos ellos.

Por otra parte, en lo que respecta a la temática el presente texto, totalmente consagrado a la transmisión de conocimientos teóricos, aborda los sistemas de encendido e inyección de motores tan en auge en la actualidad. Como se desprende de las expresiones está dirigido fundamentalmente a especialistas y estudiosos en mecánica de automóviles, quienes serán capaces de comprender el texto en su plenitud, aunque el contenido general no escapa al entendimiento de los iniciados en esta práctica. La estructura externa presenta tres párrafos bien delimitados que corresponden a tres sistemas de motores diferentes. Dado que nuestro quehacer en esta publicación se centra en el estudio lingüístico de la terminología automovilística, hacemos acopio al final de cada párrafo de los vocablos más determinantes de cada sistema de inyección que se corresponden con las partes esenciales de los sistemas mencionados. Además, nos vemos abocados a renunciar a la exposición de medios 
extralingüísticos (Fluck, 1985: 210), es decir, a todo tipo de ilustraciones por imperativos de espacio, aunque sí es cierto que cualquier ilustración, diagrama, plano o dibujo que acompañe al código lingüístico, como figura en el texto original, es una firme ayuda en aras de facilitar la comprensión al lector y, por ende, al traductor técnico.

En el aspecto estilístico se caracteriza por ser un texto referencial, puramente informativo carente de cualquier función estética y retórica, cumpliendo así los cánones de la precisión y el rigor en los datos que impera en el lenguaje técnico. En general, los textos técnicos responden a cuestiones meramente referenciales, cuya finalidad es la exposición de un contenido temático con una intención informativa o ilustrativa. El lenguaje técnico empleado es, a todas luces, denotativo sin ningún tipo de reflexiones subjetivas y matices personales en cuanto a estilo y contenido, destacando por encima de todo una exposición simple y, a su vez, rigurosa y lógica.

Texto:

\section{MODERNE ZÜND- UND EINSPRITZSYSTEME}

\section{AN OTTO MOTOREN}

KE-Jetronic

Die KE-Jetronic ist ein Benzineinspritz-System, bei dem die Grundfunktionen mechanischhydraulisch und die Zusatzfunktionen elektronisch gesteuert werden. Unterschieden werden die Versionen KE 1-5, bei Audi gibt es eine KE-Motronic. Die Kombination von Mechanik und Elektronik bietet Vorteile bei defekter Elektronik (Notlauf), hat aber Nachteile auf der Kostenseite.

Die KE-Jetronic gliedert sich in 4 Funktionsbereiche:

- Luftmengenmessung nach dem Stauscheibenprinzip.

- Steuerung der Kraftstoffmenge für Grundfunktionen.

- Kontinuierliche Benzineinspritzung.

- Elektronische Steuerung der Gemischanreicherung für Kaltstart, Warmlauf, Beschleunigung und Vollast.

1) Einspritzventile

2) Druckregler

3) Kaltstartventil

4) Potentiometer
5)Leerlaufsteller

6)Leerlaufschalter

7) elektro-hydraulischer Drucksteller

\section{KE-Motronic}

Die KE-Motronic (Audi) besteht aus einer KE-Jetronic und einer elektronischen Zündanlage mit Klopfsensor und rotierendem Hochspannungsverteiler. Das System enthält eine LambdaRegelung mit Katalysator und einen Aktivkohlebehälter für die Kraftstoffdämpfe. 


\section{Zündungsendstufe}
1) Zündspule
10) Startventil
2) Drehzahlgeber
11) Drehsteller
3) Lambda-Sonde
12) Speicher
4) NTC (negativer Temperatur-Koeffizient)
13) Aktivkolebehälter
5) Hochspannungsverteiler
14) Gemischregler
6) Klopfsensor
15) Regler
7) Lastschalter
16) Filter
8) Steuergerät
17) Pumpe
9) Tankentlüftungsventil
18) Drucksteller

\section{L-Jetronic}

Die L-Jetronic Systeme (L- LE- LU- L3- LH) sind elektronisch gesteuerte Benzineinspritzsysteme mit Luftmengen- bzw. -Luftmangenmessung. Der Kraftstoff wird in Abhängigkeit von der angesaugten Luftmenge bzw. Luftmasse und der Motordrezahl zugemessen und intermittierend eingespritzt. Die elektronische Verarbeitung der Sensordaten ergibt eine optimale Anpassung des Luft-Kraftstoff-Verhältnisses an die Erfordernisse des Motors.

\section{Systemübersicht}
1) Kraftstoff-Behälter
10) Zündspüle
2) Pumpe
11) Steuer-Relais
3) Filter
12) Steuergerät
4) Verteiler
13) Luftmengenmesser
5) Druckregler
14) Drosseklappen-Schalter
6) Einspritzventil
15) Motor-Temperatursensor
7) Kaltstartventil
16) Zündschalter
8) Thermo-Zeit-Schalter
17) Leerlauf-Einstellschraube
9) Zusatz-Luftschieber
18) CO-Einstellschraube

\section{Nivel léxico-semántico.}

En lo que se refiere al léxico del texto -principal característica del lenguaje especializado, puesto que no suele tener un ámbito más amplio de aplicación que el creado por la misma comunidad de especialistas-, sí predomina el vocabulario técnico de dominio general, ya que reúne términos que están al alcance de cualquier profano en la materia por haberse consolidado su uso en la lengua común, pongamos como ejemplo: Benzineinspritzung, Katalysator o Zündanlage, incluyendo el vocablo System, cuya aplicación, según la especialidad en la que aparece, resulta enormemente extensa. Todo ello nos corrobora que, en muchos casos, resulta imposible establecer una línea divisoria entre el lenguaje común y el lenguaje especializado, pues no cabe la menor duda de que este último se cimenta sobre los soportes de la lengua común. 
Por otra parte, aparecen los términos exclusivamente técnicos que están al servicio de las personas que ejercen una actividad especializada y, en este caso, este campo semántico delimitado introduce un léxico ajeno a los que no se dedican a la práctica de la automoción, es decir, léxico especializado que destaca por su significado unívoco y específico. Citamos a continuación, algunos de estos términos especializados que recubren nociones eminentemente mecánicas como Stauscheibenprinzip, Klopfsensor, Aktivkohlebehälter entre otros. Todos ellos tecnicismos que tratan de definir con la mayor precisión el fenómeno que designan y, en consecuencia, delimitan más el entendimiento del texto. Como apunta Hohnhold (1982:1) los tecnicismos son "Wörter mit (möglichst) genau abgegrenzter, (möglichst) verbindlich festgelegter und (möglichst) bekanntgemachter Bedeutung". Por consiguiente, los términos técnicos son palabras que corresponden a una noción perfectamente definida.

También destacamos, a pesar de la limitada extensión del texto, la frecuente tendencia a sustantivar los términos que derivan de los verbos acabados en -ung como Regelung, Verarbeitung o Anpassung.

Mención aparte merece la denominación Lambda-Regelung, donde percibimos la fuerte vinculación que se mantiene con la lengua griega al incorporar la letra de su abecedario sin ninguna merma en su naturaleza. Señala García Yebra (1987:83) la copiosa cantidad existente de híbridos de préstamo y calco o viceversa, donde la lengua alemana se combina con el griego y el latín. Y es que, aún hoy en día, el legado lingüístico de base griega y latina continúa siendo una fuente suculenta de tecnicismos con la correspondiente adaptación de los vocablos a la lengua alemana. Pero junto a esta reminiscencia grecolatina, existe en el vocabulario técnico otra influencia más actual, la de la lengua inglesa. Así, pongamos como ejemplo: Kaltstart. Por tanto, llegamos a la conclusión de que los lenguajes especializados amplian constantemente los límites de cualquier lengua en buena medida debido a la internacionalización de nuestra era y todo ello va directamente en función de la cantidad de campos especializados que existan.

Por lo que se refiere a la formación de compuestos, uno de los procedimientos más importantes en la creación de palabras nuevas, especialmente en la lengua alemana, el sustantivo acapara el peso específico en la composición. El empleo reiterado de términos compuestos como Luftmasse y Sensordaten, resultado de la suma de dos sustantivos, se manifiesta como la fórmula más empleada. Asimismo, se aprecian palabras compuestas que fusionan dos sustantivos que derivan de verbos como Motordrehzahl. Y otras formaciones que constan de prefijo más verbo, que construyen verbos de distinto significado a partir de verbos simples, tal es el caso de einspritzen.

Por otra parte, la claridad de exposición se apoya en verbos como bestehen aus, que manifiesta con lógica lo que se va a decir a continuación, y especialmente el verbo sein, muy útil para iniciar toda clase de definiciones. Sin embargo, cabe constatar la escasa relevancia significativa del verbo con respecto a la significación sustantiva, sobre todo, por la escasa aportación de verbos que pertenezcan al campo terminológico como ejemplo citamos: einspritzen.

El adjetivo tiene un uso restrictivo en el texto. Se evitan todo tipo de adjetivos valorativos en un discurso escrito que se caracteriza por la objetividad, citamos: optimale Anpassung, donde el adjetivo se muestra para precisar y matizar. Por lo demás, aparece el participio con función de adjetivo antepuesto al nombre: rotierendem Hochspannungsverteiler. 


\section{Nivel morfosintáctico.}

En cuanto al plano de la sintaxis, procedamos a analizar brevemente algunas peculiaridades que se producen en este nivel. Observamos el dominio de oraciones enunciativas simples frente a las oraciones compuestas. Se caracterizan en líneas generales por la claridad y la exactitud, imponiendo una sencillez expositiva con total ausencia del más mínimo artificio, aunque a veces la exposición de datos complicados en el discurso escrito presenta una complicada configuración sintáctica que inevitablemente conduce a obstaculizar el entendimiento de la información.

Por lo que respecta el modo verbal impera el indicativo que contribuye a la objetividad y claridad de los textos técnicos. Entre las formas de tiempo verbal más generalizada corresponde el dominio claramente al presente con valor actual, recurrriendo a las oraciones en pasiva, rasgo esencial del lenguaje técnico, que subrayan e intensifican la objetividad y acentúan la expresión de la impersonalidad para apartar toda referencia personal al autor del texto.

En las formas personales destaca el empleo prioritario y la presencia casi absoluta de la tercera persona del singular como una muestra más de la objetividad seguida de la tercera persona en plural con total ausencia de la primera persona como referencia al autor del escrito.

Observamos también el uso de siglas, que remedian un estilo recargado en los escritos técnicos. Como ejemplo podemos citar: NTC y CO.

Traducción del texto

SISTEMAS MODERNOS DE ENCENDIDO E INYECCIÓN

EN MOTORES OTTO

KE-Jetronic

El KE-Jetronic es un sistema de inyección de gasolina, en el que las funciones principales se dirigen de forma mecánica e hidráulica y las funciones complementarias de forma electrónica. Se diferencian las versiones KE 1-5. Audi dispone del sistema KE-Motronic. La combinación de mecánica y electrónica ofrece ventajas ante una electrónica defectuosa (situación de emergencia), pero desventajas en lo que al coste se refiere.

El sistema KE-Jetronic se divide en cuatro campos de funcionamiento:

- Medición de la masa de aire según el principio de plato sonda.

- Dirección de la cantidad de combustible para las funciones principales.

- Inyección de gasolina continua.

- Control electrónico de la mezcla para el arranque en frío, el calentamiento, la aceleración y la plena carga.
1) Válvulas de inyección
5) Estabilizador de ralentí
2) Regulador de presión
6) Conmutador de ralentí
3) Inyector
7) Dispositivo electrohidráulico de presión
4) Potenciómetro 


\section{KE-Motronic}

El sistema KE-Motronic (Audi) está compuesto de un sistema KE-Jetronic y un sistema de encendido electrónico con sensor de picado y distribuidor rotativo de alta tensión. El sistema contiene una regulación Lambda con catalizador y un recipiente de carbón activo para los gases de combustible.

Fase final del encendido
1) Bobina de encendido
10) Inyector de arranque
2) Transmisor de revoluciones
11) Válvula de aire adicional
3) Sonda Lambda
12) Acumulador de presión
4) Coeficiente de temperatura negativo
13) Recipiente de carbón activo
5) Distribuidor de alta tensión
14) Regulador de mezcla
6) Sensor de picado
15) Regulador
7) Conmutador de mariposa
16) Filtro
8) Unidad de control
17) Bomba
9) Tanque de la válvula de aire
18) Regulador de presión

\section{L-Jetronic}

Los sistemas L-Jetronic (L- LE- LU- L3- LH) son sistemas de inyección de gasolina con medición de la masa de aire dirigidos electrónicamente. El combustible se distribuye dependiendo de la masa de aire admitida y de las revoluciones del motor, y es inyectado de forma intermitente. La elaboración electrónica de los datos del sensor da por resultado un ajuste óptimo de combustible y aire ante las exigencias del motor.

\section{Cuadro general del sistema}
1) Depósito de combustible
10) Bobina de encendido
2) Bomba
11) Relé de inyección
3) Filtro
12) Unidad de control
4) Delco
13) Caudalímetro
5) Regulador de presión
14) Conmutador de la válvula de mariposa
6) Válvula de inyección
15) Sensor de temperatura del motor
7) Inyector
16) Llave de contacto
8) Termocontacto temporizado
17) Tornillo de regulación de ralentí
9) Válvula de aire adicional
18) Tornillo de regulación de $\mathrm{CO}$

\section{A MODO DE CONCLUSIÓN}

Para terminar, unas últimas palabras con las que es nuestra intención constatar que debido al constante crecimiento del sector automovilístico, se aprecian últimamente signos 
esperanzadores, que auguran un posible final a la sequía investigadora que padece la lengua alemana en este campo del saber. Las circunstancias actuales nos permiten pensar en un futuro más alentador, en el que los estudiosos de las lenguas técnicas, encuadrado en este área de conocimiento, aporten su grano de arena e impulsen, de esta manera, el estudio del alemán técnico referido a la automoción y, en consecuencia, promuevan y promocionen su investigación.

Por último, el objetivo que hemos pretendido alcanzar mediante esta aproximación al lenguaje técnico alemán en el campo de la automoción no ha sido otro que intentar formular una definición relativamente compacta y contundente en aras de justificar la cuestión planteada en la presente comunicación. En virtud de todo ello, estamos convencidos de haber expuesto elementos de juicio suficientes como para poder defender la existencia de un lenguaje especializado en torno al sector turístico alemán.

No cabe la menor duda que el lenguaje técnico referido a la automoción es un campo relativamente virgen en cuanto a la investigación lingüística se refiere, dada la corta historia del automóvil, a pesar de las cuantiosas publicaciones de todo tipo de manuales de mecánica o revistas de publicidad, no exentas de características y comentarios técnicos, que empresas alemanas como BOSCH, BMW o VW ponen a disposición a los concesionarios para la venta de materiales y vehículos. Y es que, obviamente, este tipo de lenguaje especializado no ha alcanzado aún la relevancia y autonomía propia como sucede con el lenguaje jurídico, administrativo, económico o publicitario. Sólo puede ser considerado un subgrupo o una ramificación de lo que entendemos por lenguaje técnico, ya que abarca buena cantidad de sus características, tan comunes a otros lenguajes temáticos integrados en el ámbito de la técnica. Sin embargo, el alto grado de especialización que ofrece el lenguaje de la automoción y la relevancia que presenta por sí mismo le situarán en un futuro no muy remoto entre los lenguajes técnicos de mayor interés e identidad.

En definitiva, se trata de un lenguaje dirigido a lectores especializados o profanos con la intención de potenciar sus conocimientos sobre la mecánica de automóviles, no exentos de tecnicismos de difícil acceso para los no iniciados, pero que en otros casos, ofrece exposiciones simples que juegan a favor de la claridad garantizando la comprensión a cualquier lector.

\section{BIBLIOGRAFÍA}

Autofachmann 7-2 (1998), págs. 21-23.

ARNZT, R./H. Picht (1995), Introducción a la terminología, Pirámide, Madrid.

BACHMANN, R. (1992), "Übersetzen technischer Fachtexte. Was muß man können? Wie kann man es lernen? Ein Beitrag aus praktischer und didaktischer Sicht”. En: Lebende Sprachen 4, págs. 145-151.

BEIER, R. (1979), "Zur Syntax in Fachtexten”. En: Mentrup, W. (ed.), Fachsprachen und Gemeinsprache (=Sprache der Gegenwart 46)Z. Jahrbuch des Instituts für deutsche Sprache, Pädagogischer Verlag Schwann, Düsseldorf, págs. 276-301.

FLUCK, H.-R. (1985), Fachsprachen, Francke Verlag, München.

GARCÍA YEBRA, V. (1987), "Préstamo y calco en español y alemán. Su interés lingüístico y su tratamiento en la traducción", en Problemas de la traducción, Fundación "Alfonso X El Sabio", Madrid, págs. 75-89. 
HAHN, W. Von (1980), "Fachsprachen", en Althaus, H.P./Henne, H./Wiegand, H.E. (eds.), Lexikon der Germanistischen Linguistik, Niemeyer Verlag, Tübingen, págs.390395.

HOFFMANN, L. (1985), Kommunikationsmittel Fachsprache. Eine Einführung, Narr, Tübingen.

HOHNHOLD, I. (1982), "Grundbegriffe im Bereich und im Umfeld übersetzungsorienter Terminologiearbeit”, en Lebende Sprachen 1, págs. 1-5.

MAILLOT, J. (1997), La traducción científica-técnica, Gredos, Madrid.

MARTÍN, J. et al. (1996), Los lenguajes especiales. Lenguaje jurídico-administrativo. Lenguaje científico-técnico.Lenguaje humanístico. Lenguaje periodístico y publicitario. Lenguaje literario, Ed. Comares, Granada.

MINK, H. (1980), Diccionario técnico (Alemán-Español), Herder, Barcelona.

NEWMARK, P. (1995), Manual de traducción, Cátedra, Madrid.

PUNKKI - ROSCHER, M. (1995), Nominalstil in populärwissenschaftlichen Texten: zur Syntax und Semantik der komplexen Nominalphrasen, Peter Lang, Frankfurt am Main.

RADDE, K.H. (1976), Polytechnisches Wörterbuch, VEB Technik, Berlin.

ROELCKE, T. (1999), Fachsprachen, Erich Schmidt Verlag, Berlin.

SLABY, R.J. / GROSSMANN, R. (1975), Wörterbuch der spanischen und deutschen Sprache, Brandstetter, Wiesbaden.

SPIEGEL, H.-R. (1979), "Neubenennungen in den technischen Fachsprachen. Bestandaufnahme und Entwicklungtendenzen", en Der Deutschunterricht 5, págs. 2234. 
